\title{
Higher-order $O\left(\alpha^{2}\right)$ and $O\left(\alpha \alpha_{s}\right)$ corrections to $\sigma_{\text {tot }}\left(e^{+} e^{-} \rightarrow\right.$ hadrons $)$ and $Z$-boson decay rate
}

\author{
A.L. Kataev ${ }^{1}$ \\ Theory Division, CERN, CH-1211 Geneva 23, Switzerland
}

\begin{abstract}
We calculate analytically the $O\left(\alpha^{2}\right)$ and $O\left(\alpha \alpha_{s}\right)$ corrections to the final states in the processes $e^{+} e^{-} \rightarrow$ hadrons and $Z^{0} \rightarrow$ hadrons, and estimate their contributions at the $Z^{0}$ pole.
\end{abstract}

CERN-TH.6465/92

April 1992

\footnotetext{
${ }^{1}$ On leave of absence from the Institute for Nuclear Research, Moscow 117312, Russia. Bitnet: KATAEV@CERNVM
} 

1. The determination of the strong coupling constant $\alpha_{s}$ at different energies is one of the main outcomes of the modern perturbative QCD (see e.g. refs. [1], [2]). The precise analysis of the QCD perturbative contributions to the characteristics of various processes enables one to verify the property of running of $\alpha_{s}$ and to express, if one wishes, the results extracted for $\alpha_{s}$ in terms of the conventional QCD scale parameter $\Lambda_{\overline{M S}}$ so as to verify its process-independence and flavour-dependence.

The characteristics of the $e^{+} e^{-} \rightarrow$ hadrons and $Z^{0} \rightarrow$ hadrons processes are among the most deeply studied quantities from these points of view (see e.g. [2]-[5]). The experimental basis for the determination of $\alpha_{s}$ is provided by the continuous accumulation of experimental data from the TRISTAN, LEP, etc., $e^{+} e^{-}$colliders. The perturbative QCD results include massless nextto-leading-order (NL) QCD corrections to $\sigma_{t o t}\left(e^{+} e^{-} \rightarrow\right.$ hadrons) [6], [7] and $\Gamma\left(Z^{0} \rightarrow\right.$ hadrons $)$, the recently calculated next-next-to-leading-order (NNL) terms[8], and the $O\left(\mathrm{~m}^{2} / \mathrm{s}\right)$ corrections to these quantities, which were also calculated at the NNL level [9], [10], starting from the results of refs. [11], [12]. The theoretical meaning of the higher-order massless $\alpha_{s}$ corrections is that the $O\left(\alpha_{s}^{2}\right)$ term fixes the scale (or the scheme) of the definition of the QCD parameter $\Lambda$ whilst the NNL term estimates the error of truncation of the considered asymptotic series in the QCD coupling constant.

It should be stressed that taking into account the negative (!) massless $\overline{M S}$ scheme $O\left(\alpha_{s}^{3}\right)$ correction to $R(s)=\sigma_{t o t}\left(e^{+} e^{-} \rightarrow\right.$ hadrons $) / \sigma\left(e^{+} e^{-} \rightarrow \mu^{+} \mu^{-}\right)$[8], and therefore to the related quantity $\Gamma\left(Z^{0} \rightarrow\right.$ hadrons $)$, increases the values of $\Lambda_{\overline{M S}}$ extracted from $R(s)$ and $\Gamma\left(Z^{0} \rightarrow\right.$ hadrons $)$ data [3]-[5] (for the recent analysis of the $R(s)$ QCD predictions, see ref. [13]). This in turn leads to the discrepancy between the values of $\alpha_{s}\left(M_{Z}\right)$ (and thus $\Lambda_{\overline{M S}}$ ) extracted at the NNL level from the analysis of the total hadronic cross-section data and $\Gamma\left(Z^{0} \rightarrow\right.$ hadrons $)$ and the similar results obtained at the NL QCD level from other processes, say deep-inelastic scattering, $e^{+} e^{-} \rightarrow$ jets, etc. [14], [13], [15]. Indeed, the value $\alpha_{s}\left(M_{Z}\right)=0.133 \pm 0.012$ extracted at the NNL level from LEP data on $\Gamma\left(Z^{0} \rightarrow\right.$ hadrons $)$ value is larger than the result, transformed to the $Z^{0}$-pole, of the NL QCD analysis of the BCDMS and SLAC deep-inelastic scattering data, namely $\alpha_{s}\left(M_{Z}\right)=0.113 \pm 0.005[15]$, or the result obtained from the quarkonia decays, namely $\alpha_{s}\left(M_{Z}\right)=0.113 \pm_{0.005}^{0.007}[15]$. Note, however, that this discrepancy is within possible statistical experimental uncertainties.

It should be stressed, as was first pointed out by Yndurain[16], that the electromagnetic $O(\alpha)$ corrections can play an important role in the analysis of the $e^{+} e^{-}$data. In this work, in order to understand whether taking account of the higher- order electromagnetic corrections could help resolve the above- discussed discrepancies, we calculate the $O\left(\alpha^{2}\right)$ and $O\left(\alpha \alpha_{s}\right)$ contributions to $R(s)$ and $\Gamma\left(Z^{0} \rightarrow b \bar{b} \rightarrow\right.$ hadrons $)$ from the photon emission in the final states and estimate their values at the $Z^{0}$ pole.

2. It is known that from the number of background-corrected hadronic events $N_{e v}$ the $R(s)$ value is determined from the expression

$$
R=\frac{N_{e v}}{\epsilon(1+\delta) L} \frac{1}{\sigma_{\mu \mu}^{0}}
$$

where $L$ denotes the integrated luminosity of the analysed data sample, $\epsilon$ is the acceptance of the applied-event selection procedure and $\sigma_{\mu \mu}^{0}=4 \pi \alpha^{2} / 3 s$ is the Born cross-section for $\mu$-pair production. The radiative corrections to the reaction $e^{+} e^{-} \rightarrow q \bar{q} \rightarrow$ hadrons are taken into account by the factor $(1+\delta)$, which relates the Born cross-section $\sigma^{0}$ for hadron production to the measured cross-section

$$
\sigma\left(e^{+} e^{-} \rightarrow \text { hadrons }\right)=\sigma^{0}(1+\delta)=\sigma^{0}\left(1+\delta_{\text {soft }}+\delta_{\text {hard }}+\delta_{\text {vertex }}+\delta_{\text {vac }}\right)
$$

This correction term receives contributions from soft and hard initial-state radiation, from initial vertex corrections, and from vacuum polarization terms with lepton $(e, \mu, \tau)$ and quark loops. The 
higher-order radiative corrections to the initial states have been calculated in ref. [17] (for detailed discussion of these contributions as well as of other related effects, see the extensive review [18]).

The lepton vacuum polarization terms are usually taken into account in the form of the one-loop on-shell (OS) scheme approximation for the QED-invariant charge (see e.g. [19]). This procedure turns out to be almost non-sensitive to whether higher-order logarithmic electromagnetic contributions to the photon vacuum polarization function calculated in ref.[20] at the four-loop level are taken into account ${ }^{2}$. As to the quark-loops effects, they are usually taken into account through the dispersion relation (see e.g. [21]):

$$
\delta_{v a c}^{q}=-2 \operatorname{Re} \Pi_{\mathbf{h}}(\mathrm{s})
$$

with

$$
\Pi_{h}(s)=\frac{\alpha s}{3} \int_{4 m_{\pi}^{2}}^{\infty} \frac{R\left(s^{\prime}\right)}{s^{\prime}\left(s-s^{\prime}\right)} d s^{\prime}
$$

saturated by the experimental data $R^{\exp }\left(s^{\prime}\right)$ at low and intermediate energies. At higher energies, $R\left(s^{\prime}\right)$ is approximated by its theoretical expression.

However, there is also another place where QED and QCD radiative corrections to the photon vacuum polarization function come into play. Indeed, the theoretical expression for $R(s)$ is related to the imaginary part of the one-particle irreducible diagrams, contributing to the photon vacuum polarization function, with external quark loops

$$
R(s) \sim \operatorname{Im}_{\mathrm{q}}\left(\mathrm{x}_{\mathrm{n}}, \mathrm{x}_{\mathrm{f}}, \alpha, \alpha_{\mathrm{s}}\right),
$$

where $x_{n}=-q^{2} / m_{n}^{2}, x_{f}=-q^{2} / m_{f}^{2}, m_{n}(1 \leq n \leq N)$ are the masses of $N$ leptons propagating in the internal loops of the corresponding three-loop diagrams and $m_{f}(1 \leq f \leq F)$ are the masses of $F$ quarks flavours.

The pure-QCD contributions to $R(s)$ are known at present at the NNL level, which corresponds to the four-loop QCD approximation of the photon vacuum polarization function $\Pi_{q}$ [8]. Let us now calculate the pure electromagnetic and the mixed QED-QCD contributions to $R(s)$ from the three-loop expression of $\Pi_{q}$. This can be done using the diagram-by-diagram results of ref. [7]. The final analytical expression we are interested in reads

$$
\begin{aligned}
R^{E M}(s)= & 3 \sum_{f=1}^{F} Q_{f}^{2}\left\{\frac{3}{4} Q_{f}^{2} \frac{\alpha}{\pi}+\left[-\frac{3}{32} Q_{f}^{4}-\left(\frac{11}{8}-\zeta(3)\right) Q_{f}^{2}\left(N+3 \sum_{j=1}^{F} Q_{j}^{2}\right)\right.\right. \\
& \left.+\frac{1}{4} Q_{f}^{2}\left(\sum_{i=e, \mu, \tau} \ln \left(\frac{s}{m_{i}^{2}}\right)+3 \sum_{j=1}^{F} Q_{j}^{2} \ln \left(\frac{s}{m_{j}^{2}}\right)\right)\right]\left(\frac{\alpha}{\pi}\right)^{2} \\
& \left.-\frac{1}{8} Q_{f}^{2} \frac{\alpha}{\pi} \frac{\alpha_{s}(s)}{\pi}+O\left(\alpha^{3}\right)+O\left(\alpha^{2} \alpha_{s}\right)+O\left(\alpha \alpha_{s}^{2}\right)\right\},
\end{aligned}
$$

where $Q_{f}$ are the charges of quarks propagating in the external quark loops of the propagator-like diagrams, $Q_{j}$ are of those propagating in the internal quark loops of the corresponding three-loop diagrams, $N=3, \alpha$ is the QED coupling constant in the OS scheme (namely the fine coupling constant), and $\alpha_{s}(s)$ refer to the $\overline{M S}$ scheme. Note that the third and the fourth terms in the square brackets, which contain lepton and quark mass dependence, can be absorbed into the leading $O(\alpha)$ term if, instead of the OS scheme coupling constant one will use the one-loop approximation of the electromagnetic running coupling constant defined as

$$
\alpha(s)=\frac{\alpha}{1-\beta_{0}\left(\sum_{i=e}^{\tau} \ln x_{i}+3 \sum_{j=1}^{F} Q_{j}^{2} \ln x_{j}\right)(\alpha / \pi)}
$$

where $\beta_{0}=1 / 3$ is the one-loop coefficient of the standard QED $\beta$ function.

\footnotetext{
${ }^{2}$ This fact has been checked at our request by J. Fujimoto.
} 
3. The results obtained can be straightforwardly generalized to the case of $Z^{0} \rightarrow$ hadrons decay. To be more precise, we will consider the decay $Z^{0} \rightarrow b \bar{b} \rightarrow$ hadrons. Its total decay width has the following form:

$$
\begin{aligned}
\Gamma\left(Z^{0} \rightarrow \bar{b} b \rightarrow \text { hadrons }\right)= & \alpha M_{Z}\left(v_{b}^{2}+a_{b}^{2}\right) \delta^{Q E D}\left(\alpha, \alpha_{s}\right)+\alpha M_{Z}\left(v_{b}^{2}+a_{b}^{2}\right) \delta_{1}^{Q C D}\left(\alpha_{s}\right) \\
& +\alpha M_{Z} v_{b}^{2} \delta_{2}^{Q C D}\left(\frac{m_{b}^{2}}{M_{Z}^{2}}, \alpha_{s}\right)+\alpha M_{Z} a_{b}^{2} \delta_{3}^{Q C D}\left(\frac{m_{b}^{2}}{M_{Z}^{2}}, \alpha_{s}\right)
\end{aligned}
$$

where $v_{b}=\left(I_{b}^{3}-2 Q_{b} \sin ^{2} \theta_{W}\right) / 2 \sin \theta_{W} \cos \theta_{W}, a_{b}=I_{b}^{3} / 2 \sin \theta_{W} \cos \theta_{W}$ and $I_{b}^{3}=-1 / 2, Q_{b}=-1 / 3$. The function $\delta^{Q E D}\left(\alpha, \alpha_{s}\right)$ is the contribution of the electromagnetic corrections we are interested in. The QCD contribution $\delta_{1}^{Q C D}\left(\alpha_{s}\right)$ can be obtained from the results of ref. [8]. In the $\overline{M S}$ scheme it reads

$$
\delta_{1}^{Q C D}=1+\frac{\overline{\alpha_{s}}}{\pi}+1.41\left(\overline{\frac{\alpha_{s}}{\pi}}\right)^{2}-12.8\left(\overline{\frac{\alpha_{s}}{\pi}}\right)^{3}
$$

where $\overline{\alpha_{s}}=\alpha_{s}\left(M_{Z}\right)$. The massive corrections to the vector channel have been obtained in ref. [9] from the results of ref. [11]. They have the following form [9]:

$$
\delta_{2}^{Q C D}=12 \frac{\bar{m}_{b}^{2}}{M_{Z}^{2}} \frac{\overline{\alpha_{s}}}{\pi}\left[1+8.7 \frac{\overline{\alpha_{s}}}{\pi}+45.3\left(\frac{\overline{\alpha_{s}}}{\pi}\right)^{2}\right]
$$

where $\bar{m}_{b}=m_{b}\left(M_{Z}\right)$ is the renormalized in the $\overline{M S}$ scheme $b$-quark mass normalized at the $Z^{0}$ pole. The similar contributions to the axial channel were calculated recently [10] using the results of ref. [12]. They read:

$$
\delta_{3}^{Q C D}=-6 \frac{\bar{m}_{b}^{2}}{M_{Z}^{2}}\left[1+3.67 \frac{\overline{\alpha_{s}}}{\pi}+14.29\left(\frac{\bar{\alpha}}{\pi}\right)^{2}\right] .
$$

Let us estimate the numerical value of the perturbative QCD contributions at the $Z^{0}$ pole. As input parameters we will use the values $\overline{\alpha_{s}}=\alpha_{s}\left(M_{Z}\right)=0.133, M_{Z}=91.2 \mathrm{GeV}$, and $\bar{m}_{b}=m_{b}\left(M_{Z}\right)=2.7$ $\mathrm{GeV},[10]$ which corresponds to the $b$-quark pole mass $m_{b}=4.7 \mathrm{GeV}$. Then we have

$$
\begin{aligned}
& \delta_{1}^{Q C D}=1+0.0423+0.0025-0.0001=1+4.47 \times 10^{-2} \\
& \delta_{2}^{Q C D}=0.0004[1+0.37+0.08]=5.8 \times 10^{-4} \\
& \delta_{3}^{Q C D}=-0.0052[1+0.16+0.03]=-6.2 \times 10^{-3}
\end{aligned}
$$

The contributions of the calculated electromagnetic corrections have the following form

$$
\begin{gathered}
\delta^{Q E D}=\frac{1}{12} \frac{\alpha}{\pi}+\left[-0.13+\frac{1}{36}\left(\ln \frac{M_{Z}^{2}}{m_{e}^{2}}+\ln \frac{M_{Z}^{2}}{m_{\mu}^{2}}+\ln \frac{M_{Z}^{2}}{m_{\tau}^{2}}+\right.\right. \\
\left.\left.+\frac{4}{3} \ln \frac{M_{Z}^{2}}{m_{u}^{2}}+\frac{1}{3} \ln \frac{M_{Z}^{2}}{m_{d}^{2}}+\frac{1}{3} \ln \frac{M_{Z}^{2}}{m_{s}^{2}}+\frac{4}{3} \ln \frac{M_{Z}^{2}}{m_{c}^{2}}+\frac{1}{3} \ln \frac{M_{Z}^{2}}{m_{b}^{2}}\right)\right]\left(\frac{\alpha}{\pi}\right)^{2}-\frac{1}{18} \frac{\alpha}{\pi} \frac{\overline{\alpha_{s}}}{\pi} .
\end{gathered}
$$

For the rough estimates we transform the normalized at $1 \mathrm{GeV} \overline{M S}$ scheme running masses of light quarks $m_{u}=0.005 \mathrm{GeV}, m_{d}=0.01 \mathrm{GeV}, m_{s}=0.18 \mathrm{GeV}[22]$ to the pole masses and take $m_{c}=1.5$ $\mathrm{GeV}, m_{b}=4.7 \mathrm{GeV}$, and the table values of lepton masses. As a result we obtain

$$
\delta^{Q E D}=1.9 \times 10^{-4}+1.3 \times 10^{-5}-5.5 \times 10^{-6},
$$

where the $\alpha \alpha_{s}$ contribution is larger then the order $\alpha^{2}$ constant term. Therefore, the dominating electromagnetic contribution comes from the previously known term, (leading in $\alpha$ ) which is usually included in the precise analysis of experimental data. A similar result is expected for the $\tau \rightarrow$ 
$\nu_{\tau}+$ hadrons decay, where the leading electromagnetic corrections were calculated in ref. [23] and summarized in ref. [24].

As to the value of the NL $\alpha^{2}$ term, it mainly comes from the logarithmic contributions responsible for the effect of running of the electromagnetic coupling constant. Notice in these circumstances the importance of taking into account the relatively sizeable quark- mass-dependent terms. Higher-order $O\left(\alpha^{3}\right), O\left(\alpha^{2} \alpha_{s}\right)$ and $O\left(\alpha \alpha_{s}^{2}\right)$ corrections to $R(s)$ and $Z$-boson decay width could be calculated, in principle, using the four-loop level diagram-by-diagram results obtained in the course of the work of ref. [8]. However, we expect that they will be small, and therefore not important either in theoretical and in the experimental analyses, at the current level of understanding.

Note also that in our discussions we did not touch the consideration of the effects of electroweak corrections analysed in detail at the one-loop level (see e.g. [25]-[28], [18]).

4. To conclude, we have shown that the NL electromagnetic corrections to the final states production in $e^{+} e^{-} \rightarrow$ hadrons and $Z^{0} \rightarrow$ hadrons processes, as evaluated by us, are small. Therefore taking them into account cannot help to resolve the discrepancies between the values of $\alpha_{s}\left(M_{Z}\right)$ extracted from different experiments [15]. More work in this direction is needed, including a more detailed analysis of different experimental data.

\section{Acknowledgements}

We are grateful to Y. Shimizu and to our colleagues from KEK, where the work was started, for their hospitality in Japan. The hospitality of the members of the Theoretical Division of CERN, where the work was finished, is gratefully acknowledged. It is a pleasure to thank G. Altarelli, J. Fujimoto and Y. Shimizu for helpful discussions. 


\section{References}

[1] G. Altarelli, Annu. Rev. Nucl. Part. Sci., 39 (1989) 357.

[2] S. Bethke and J. E. Pilcher, Univ. Heidelberg preprint HD-PY 92-06 (1992); Enrico Fermi Inst. preprint EFI 92-14 (1992); Annu. Rev. Nucl. Part. Sci., 42 (1992) to appear.

[3] G. d'Agostini, W. de Boer and G. Grindhammer, Phys. Lett. B229 (1991) 160.

[4] R. Marshall, Z. Phys. C43 (1989) 587.

[5] V. Branchina, P. Castorina, M. Consoli and D. Zappala, Phys. Rev. Lett. 65 (1990) 3237.

[6] K. G. Chetyrkin, A. L. Kataev and F. V. Tkachov, Phys. Lett. B85 (1979) 277;

M. Dine and J. Sapirstein, Phys. Rev. Lett. 43 (1979) 668;

W. Celmaster and R. J. Gonsalves, Phys. Rev. Lett. 44 (1980) 560.

[7] K. G. Chetyrkin, A. L. Kataev and F. V. Tkachov, Institute for Nuclear Research report INR P-0170 (1980) (unpublished).

[8] S. G. Gorishny, A. L. Kataev and S. A. Larin, in "Standard Model and Beyond" Proc. First International Workshop CERN-IHEP-JINR, 1-5 October 1990, eds. S. Dubnicka, D. Ebert and A. Sazonov, World Scientific, Singapore (1991) p. 288; Phys. Lett. B259 (1991) 144.

[9] K. G. Chetyrkin and J. H. Kühn, Phys. Lett B248 (1990) 359.

[10] K. G. Chetyrkin, J. H. Kühn and W. Kwiatowski, University of Karlsruhe preprint TTP 92-07 (1992) to be published.

[11] S. G. Gorishny, A. L. Kataev and S. A. Larin, Nuovo Cim. A92 (1986) 117;

L. R. Surguladze, Institute for Nuclear Research report П-0644 (1989) (unpublished).

[12] S. G. Gorishny, A. L. Kataev, S. A. Larin and L. R. Surguladze, Mod. Phys. Lett. A5 (1990) 2703; Phys. Rev. D43 (1991) 1633.

[13] J. Chyla, A. L. Kataev and S. A. Larin, Phys. Lett. B267 (1991) 269.

[14] A. L. Kataev, Univ. Michigan report UM-TH-91-04 (1991) (unpublished).

[15] S. Catani and S. Bethke, summary talk of the round-table discussion at the XXVII Recontres de Moriond: "QCD and High Energy Hadronic Interactions", 22-28 March 1992, to be published in the Proceedings ed. by J. Tran Thanh Van.

[16] F. Yndurain, Nucl. Phys. B136 (1978) 533.

[17] E. A. Kuraev and V. S. Fadin, Sov. J. Nucl. Phys. 41 (1985) 466.

[18] J. Fujimoto, M. Igarashi, N. Nakazawa, Y. Shimizu and K. Tobimatsu, Prog. Theor. Phys. Suppl. $100(1990) 1$.

[19] M. Igarashi, N. Nakazawa, T. Shimada and Y. Shimizu, Nucl. Phys. B263 (1986) 374; J. Fujimoto and Y. Shimizu, Mod. Phys. Lett. A3 (1988) 581.

[20] A. L. Kataev, KEK preprint KEK 91-183 (1991) to be published in Phys. Lett. B.

[21] F. A. Berends and G. J. Koman, Phys. Lett. B63 (1976) 432. 
[22] S. G. Gorishny, A. L. Kataev and S. A. Larin, Phys. Lett. B135 (1984) 457;

C. A. Dominguez and E. de Rafael, Ann. Phys. 174 (1987) 357.

[23] E. Braaten and C. S. Li, Phys. Rev. D42 (1990) 3888.

[24] E. Braaten, S. Narison and A. Pich, preprint CERN-TH-6070/91 (1991), to be published in Nucl. Phys. B.

[25] G. Passarino and M. Veltman, Nucl. Phys. B160 (1979) 151.

[26] D. Yu. Bardin, P. Ch. Christova and O. M. Fedorenko, Nucl. Phys. B197 (1982) 1.

[27] W. Beenaker and W. Hollik, Zeit. Phys. C40 (1988) 141.

[28] M. Consoli and W. Hollik, in Z physics at LEP1 ( CERN 89-08, Geneva, 1989) vol. 1, p. 7; eds. G. Altarelli, R. Kleiss and C. Verzegnassi . 